\title{
El alcance de las prácticas de dirección en la vida de los colaboradores: la relación entre la percepción de justicia y el conflicto trabajo-familia en las organizaciones del sur colombiano
}

DOI: https://doi.org/10.21158/01208160.n87.2019.2442

Corporación Universitaria Minuto de Dios andradenav@uniminuto.edu.co

Elías Ramírez-Plazas ${ }^{2}$ Universidad Surcolombiana Elias.ramirez@usco.edu.co

Humberto Rueda-Ramírez ${ }^{3}$ Universidad Surcolombiana humrueda@gmail.com

Fecha de recepción: 18 de marzo de 2019

Fecha de aprobación: 17 de diciembre de 2019

Cómo citar este artículo / To reference this article / Comment citer cet article / Para citar este artigo:

Andrade-Navia, J. M.; Ramírez-Plazas, E.; Rueda-Ramírez, H. (2019). El alcance de las prácticas de dirección en la vida de los colaboradores: la relación entre la percepción de justicia y el conflicto trabajo-familia en las organizaciones del sur colombiano. Revista Escuela de Administración de Negocios, (87), 27-41. DOI: https://doi.org/10.21158/01208160.n87.2019.2442

\section{Resumen}

El presente estudio evalúa la relación entre la justicia organizacional, en sus dimensiones de justicia procedimental y justicia interaccional, y el conflicto trabajo-familia en las empresas colombianas. La investigación fue de carácter explicativo-causal y con el fin de evaluar las relaciones y los efectos entre las variables se utilizó un modelo de ecuaciones estructurales. La investigación se realizó en tres organizaciones pertenecientes a diferentes sectores económicos — servicios, financiero y producción—, en las que se aplicaron 450 encuestas a sus empleados. En cuanto a los resultados, se obtuvo que solo la justicia interaccional presenta una relación negativa y significativa con el conflicto trabajo-familia; la relación justicia procedimental y conflicto trabajo-familia fue no significativa, además de positiva. Esta investigación y sus resultados respaldan la hipótesis de que el crecimiento empresarial, de la mano del bienestar del capital humano, depende de factores clave como la disminución de los conflictos de los empleados y la capacitación de los jefes o supervisores mediante estrategias de liderazgo transformacional que den cuenta de la justicia organizacional y de la participación activa de todos los actores, para fomentar así, relaciones y entornos laborales en los que prevalezcan valores fundamentales como el respeto, la honestidad, la dignidad y la cortesía.

Palabras clave: justicia organizacional; justicia procedimental; justicia interaccional; conflicto trabajo-familia; bienestar del capital humano; entorno laboral; liderazgo transformacional.

\footnotetext{
${ }^{1}$ Administrador de empresas y especialista en Alta Gerencia de la Universidad Surcolombiana. Magíster en Pensamiento Estratégico y Prospectiva de la Universidad Externado de Colombia. Magíster en Gerencia del Talento Humano de la Universidad de Manizales. Candidato a doctor en Agroindustria y Desarrollo Agrícola Sostenible de la Universidad Surcolombiana. ORCID: https://orcid. org/0000-0001-9644-0040

${ }^{2}$ Ingeniero industrial de la Universidad de los Andes, magíster en Economía y Administración de la Universidad Santo Tomás, magíster en Dirección Universitaria de la Universidad de los Andes y doctor en Administración de empresas de la Universidad Libre de la Empresa y de las Tecnologías de Bruselas, Bélgica. ORCID: https://orcid.org/0000-0001-8928-1547

${ }^{3}$ Licenciado en Matemáticas de la Universidad Nacional de Colombia, Contador público de la Universidad Surcolombiana, especialista en Administración de sistemas de la Universidad Nacional de Colombia y magister en Dirección Universitaria de la Universidad de los Andes. ORCID: https://orcid.org/0000-0002-9700-263X
} 


\title{
The scope of management practices in employees' lives: the relationship between the perception of justice and the work-family conflict in organizations in Southern Colombia
}

\begin{abstract}
This study evaluates the relationship between organizational justice, in its dimensions of procedural justice and interactional justice, and the work-family conflict in Colombian companies. The research uses an explanatory-causal methodology and a structural equation model in order to evaluate the relationships and effects between the variables. The research was developed in three organizations belonging to different economic sectors services, financial, and production - where 450 surveys were applied to their employees. As for the results, only the interactional justice has a negative and significant relationship with the work-family conflict; the relationship between the procedural justice and the work-family conflict was not significant, and even positive. This work and its results support the hypothesis that business growth, hand-in-hand with the well-being of human capital, depends on key factors such as the reduction of the employees' conflicts and the training of managers or supervisors by means of transformational leadership strategies that account for organizational fairness and the active participation of all the stakeholders, thus fostering working relationships and environments in which core values such as respect, honesty, dignity, and courtesy prevail.
\end{abstract}

Keywords: organizational justice; procedural justice; interactional justice; labor-family conflict; human capital welfare; work environment; transformational leadership.

\section{O escopo das práticas de gestão na vida dos funcionários: a relação entre a percepção de justiça e o conflito trabalho-família em organizações no sul da Colômbia}

\section{Resumo}

O presente estudo avalia a relação entre justiça organizacional, em suas dimensões de justiça processual e justiça interacional, e o conflito trabalho-família em empresas colombianas. A pesquisa foi de natureza explicativa-causal e com a finalidade de avaliar as relações e os efeitos entre as variáveis foi utilizado um modelo de equação estrutural. A pesquisa foi realizada em três organizações pertencentes a diferentes setores econômicos -serviços, financeiro e produção-, nas quais foram aplicadas 450 pesquisas aos seus funcionários. Quanto aos resultados, verificou-se que apenas a justiça interacional apresenta uma relação negativa e significativa com o conflito trabalho-família; a relação justiça processual e conflito trabalho-família não foram significativas, demais de positivas. Esta pesquisa e seus resultados apoiam a hipótese de que o crescimento dos negócios, em conjunto com o bem-estar do capital humano, depende de fatores-chave como a redução dos conflitos dos funcionários e o treinamento de gerentes ou supervisores por meio de estratégias de liderança transformacional que dão conta da justiça organizacional e da participação ativa de todos os atores, a fim de promover relacionamentos e ambientes de trabalho nos quais prevaleçam valores fundamentais como respeito, honestidade, dignidade e cortesia.

Palavras-chave: justiça organizacional; justiça processual; justiça interacional; conflito trabalho-família; bem-estar do capital humano; ambiente de trabalho; liderança transformacional. 
Pratiques de gestion dans la vie des collaborateurs:

$\longrightarrow$ relation entre perception de justice et conflit travail-famille dans les entreprises du sud de la Colombie

\section{Résumé}

Cette étude évalue la relation existente dans les entreprises colombiennes entre la justice organisationnelle dans ses dimensions de justice procédurale et interactionnelle avec le conflit travail-famille. L'investigation de type explicative-causale évalue et utilise les relations et les effets des variables d'un modèle d'équations structurelles. L'investigation a été réalisée dans trois entreprises appartenant à différents secteurs économiques - services, finances et production - dans lesquelles un total de 450 questionnaires ont été soumis aux employés. Les résultats obtenus montrent que seule la justice interactionnelle a une relation significative mais négative avec le conflit travail-famille; la relation entre justice procédurale et le conflit travail-famille n'étant pas significative. Cette investigation et ses résultats confirment l'hypothèse selon laquelle la croissance des entreprises et le bien-être des collaborateurs dépendent de facteurs clés tels que la réduction des conflits avec les employés, la formation des cadres et dirigeants grâce à des stratégies de leadership transformationnel rendant compte de la justice organisationnelle et de la participation active de tous les acteurs afin de favoriser les relations et l'environnement de travail dans lesquels prévalent des valeurs fondamentales telles que le respect, l'honnêteté, la dignité et la courtoisie.

Mots-clés: justice organisationnelle; justice procédurale; justice interactionnelle; conflit travail-famille; bien-être des collaborateurs; environnement de travail; leadership transformationnel. 


\section{Introducción}

$\mathrm{U}$ na de las variables que recientemente se ha vinculado al estilo de liderazgo transformacional del gerente es la «percepción de justicia organizacional», en sus dimensiones de justicia procedimental y justicia interaccional (Gillet, Fouquereau, Bonnaud-Antignac, Mokounkolo y Colombat, 2013; Taner, Turhan, Helvaci y Köprülü, 2015). En general, Greenberg (1990) define la justicia procedimental como la faceta de la justicia organizacional que se refiere a la percepción de equidad e imparcialidad en los procesos que determinan y asignan los recursos. Por su parte, la justicia interaccional corresponde a la percepción del trato que el empleado recibe justicia interpersonal- y el carácter de las explicaciones sobre las razones que fundamentan la decisión — justicia informacional- de la asignación de los resultados (Colquitt, 2001).

Así, diversos autores destacan que la justicia organizacional asume un rol mediador entre el liderazgo transformacional y los diferentes comportamientos y actitudes de los empleados. Entre estos se destacan la confianza en el líder (Zeinabadi y Rastegarpour, 2010), los comportamientos ciudadanos (Kirkman, Chen, Farh, Chen y Lowe, 2009), y el compromiso afectivo con la organización (Kim y Kim, 2015), por mencionar algunos.

Por otra parte, es de conocimiento cómo las personas que laboran en las organizaciones deben hacer frente al problema de armonizar las responsabilidades que les exige su trabajo con los deberes que demanda su familia (Williams y Alliger, 1994). Así, los empleados deben enfrentarse al dilema reiterativo de escoger entre una actividad $\mathrm{u}$ otra, con las respectivas implicaciones que dicha selección trae consigo. Este fenómeno, denominado «conflicto trabajo-familia»y en adelante CTF, es un conflicto de roles que emerge cuando las demandas de la familia se ven interferidas por los requerimientos del trabajo (Munir, Nielsen, Garde, Albertsen y Carneiro, 2012).

Dado lo anterior, el conflicto trabajo-familia ha suscitado abundante investigación desde disciplinas como, por ejemplo, la psicología, la sociología, los estudios familiares y la gestión. Tales estudios han centrado su atención en variados aspectos: el género, el tiempo yla división del trabajo, entre otros (Colquitt y Zipay, 2015; Hanif, Lambert y James, 2016; Kossek, Pichler, Bodner y Hammer, 2011).

Entre los aspectos estudiados desde la gestión se presta especial atención a la interacción entre el gerente o supervisor y su relación con los empleados; esto, en la medida en que la comunicación y el trato del jefe pueden afectar la percepción del colaborador. En este sentido, se ha investigado la percepción de justicia y su relación con el conflicto trabajo-familia (Cropanzano, Byrne, Bobocel y Rupp, 2001; Ferris, Spence, Brown y Heller, 2012). Así, por ejemplo, Judge y Colquitt (2004) encontraron que algunas dimensiones de la justicia organizacional tienen una relación negativa con el conflicto trabajo-familia exhibido por los colaboradores.

Por tanto, las investigaciones existentes relacionan la percepción de justicia organizacional con el conflicto trabajo familia - justicia organizacional $\rightarrow$ conflicto trabajo-familia-. Sin embargo, pese a haberse investigado la interacción entre tales variables, según lo que conocen los autores de este artículo, existen limitados estudios que ausculten la relación entre las dimensiones procedimental e interaccional de la justicia organizacional y el conflicto trabajo-familia. 


\section{Referentes teóricos}

\subsection{Justicia organizacional}

La justicia organizacional o JO es un constructo social acerca de lo que las personas consideran justo, más allá de su realidad objetiva. Así, la justicia organizacional es una posición personal o percepción sobre las conductas éticas y morales de la gestión en la organización (Cropanzano, Bowen y Gilliland, 2007).

Cropanzano et al. (2007) consideran que las personas se preocupan por la justicia en las organizaciones por tres razones. Primero, porque la justicia les permite predecir y controlar los resultados que, probablemente, recibirán de sus organizaciones cuando terminen una tarea con éxito (Weiss, Suckow y Cropanzano, 1999). Segundo, porque las personas desean sentirse valoradas y aceptadas en la organización; pertenecer a un grupo y sentirse relevante dentro de este es más importante, incluso, que los aspectos económicos (Tyler y Blader, 2000; Tyler y Smith, 1998). Tercero, porque las personas creen que el trato justo es la manera moral o éticamente apropiada de tratar a otros (Folger, 2001); así, llegan a reaccionar de forma negativa ante situaciones que consideran injustas.

Los estudios sobre justicia organizacional la han clasificado en tres dimensiones; justicia distributiva (Leventhal, 1976), justicia procedimental (Leventhal, 1980; Thibaut y Walker, 1975), y justicia interaccional, la cual algunos otros autores dividen en dos tipos: justicia interpersonal y justicia informacional (Greenberg, 1993). Dado que la investigación ha destacado que las dimensiones procedimental e interaccional se asocian más a los individuos que exhiben cargos de dirección y liderazgo (De Cremer, Van Dijke y Bos, 2007), a continuación se describen solo dichas dimensiones.

\subsubsection{Justicia procedimental.}

Según Greenberg (1990), la justicia procedimental o JP se refiere a la idea de equidad en los procesos que determinan y asignan los recursos en la organización. Así mismo, plantea que dicho tipo de justicia refleja los juicios de una persona sobre la imparcialidad o parcialidad del proceso de toma de decisiones de asignación de resultados. La premisa básica es que un trato justo determina las reacciones de los individuos ante las decisiones y, por tanto, es un elemento central del comportamiento de estos (Lind y Tyler, 1988).

Según las ideas de Leventhal (1980), se han planteado seis reglas que, si se siguen, generan procesos que se consideran más justos: a) la regla de consistencia, la cual establece que los procedimientos de asignación deben ser consistentes en términos de personas y tiempo; b) la regla de supresión de sesgo, en la que se declara que los intereses personales de los responsables de la toma de decisiones deben evitarse durante el proceso de asignación; c) la regla de exactitud, en relación con la bondad de la información utilizada en el proceso de asignación; d) la regla de corrección, que trata de la existencia de oportunidades para modificar decisiones injustas; e) la regla de representatividad, en la que se indica que las necesidades, los valores y las perspectivas de todas las partes afectadas por el proceso de asignación deben estar representadas en el proceso; y f) la regla de la ética, según la cual el proceso de asignación debe ser compatible con los valores morales y éticos fundamentales de los colaboradores.

\subsubsection{Justicia interaccional.}

La justicia interaccional o JI se centra en las percepciones de los individuos sobre la calidad del trato interpersonal recibido durante la toma de decisiones y los procedimientos organizacionales. Dicho tipo de justicia incluye varios comportamientos que evidencian sensibilidad social, como, por ejemplo, respeto, honestidad, dignidad y cortesía (Bies, 2001; Cropanzano, Prehar y Chen, 2002). Como se anotó, la justicia interaccional se compone de dos partes: la justicia interpersonal y la justicia 
informacional, de las cuales la primera se refiere al trato a los empleados con cortesía y respeto en la ejecución de los procedimientos y decisión frente a los resultados; la segunda, a la entrega de información a los empleados sobre las razones por las cuales se adoptaron ciertos procedimientos (Greenberg, 1990; Greenberg, 1993; Luo, 2007).

\subsection{Conflicto trabajo-familia}

Greenhaus y Beutell (1985) definieron el conflicto trabajo-familia o CTF como un tipo de conflicto entre roles, en el que las presiones o exigencias del trabajo y la familia son mutuamente incompatibles. En otras palabras, el conflicto trabajo-familia alude a aquellas situaciones en las cuales los requerimientos laborales les generan a los empleados ciertos conflictos con la familia o viceversa (Jiang, 2012). Al respecto, Greenhaus y Beutell (1985) identifican tres tipos de conflicto trabajo-familia; uno, denominado «conflicto basado en el tiempo», el cual radica en que el tiempo ocupado en un dominio disminuye la disponibilidad de este en otros roles, lo que reduce energía y crea estrés; el segundo, llamado «conflicto basado en la tensión», en el que el estrés experimentado en un rol afecta el rendimiento en el otro; y un tercer tipo, denominado «conflicto basado en el comportamiento», el cual consiste en la incompatibilidad entre varios y deseados comportamientos en los dos campos en competencia (Tziner y Sharoni, 2014).

En general, la literatura sobre conflicto trabajo-familia plantea que este tipo de conflictos pueden surgir de dos formas (Kossek y Ozeki, 1998): por una parte, el trabajo interfiere con la familia (TIF), y surge cuando las demandas laborales interfieren con las responsabilidades familiares; por otra, la familia interfiere con el trabajo (FIT) esto es, cuando las obligaciones familiares interfieren con el buen desarrollo o cumplimiento de las responsabilidades en el trabajo (Judge y Colquitt, 2004). Autores como Grandey, Cordeiro y Judd (2007), así como Kyei-Poku (2014), han hallado que el ambiente laboral suele tener un mayor nivel de interferencia en el ambiente familiar con relación al nivel de interferencia que suele tener el ambiente familiar en el laboral. Si se tiene en cuenta tal situación, la presente investigación se enfoca en la forma como la percepción de situaciones laborales, en concreto la percepción de justicia organizacional, se relaciona -interfiere- en el buen desarrollo de la vida familiar de los empleados.

\subsection{Relación entre justicia organizacional y conflicto trabajo familia}

Judge y Colquitt (2004), Greenberg y Colquitt (2005), Malisetty y Kumari (2016) y Kyei-Poku (2014) pusieron en evidencia la relación negativa entre la justicia organizacional, en sus dimensiones procedimental e interaccional, así como el conflicto trabajo-familia. En lo que corresponde a la justicia procedimental, tal relación se explica a partir de la tensión que les genera a los colaboradores percibir los procedimientos que se ejecutan como incorrectos e injustos. Por ejemplo, los empleados a quienes sus jefes no les permitían explicar por qué llegaban tarde o cometían algún error en sus trabajos, por lo general, percibían tal hecho como una injusticia, mientras que las personas escuchadas fueron más propensas a percibir el proceso como justo, incluso si los resultados no eran lo que buscaban y, finalmente, recibieron una sanción.

Por su parte, la relación con la justicia interaccional se hace evidente tanto en su faceta interpersonal como en la informacional. En la faceta interpersonal tal relación se evidencia, principalmente, cuando el supervisor se torna asequible en aspectos tales como facilitar permisos parentales y permitir horarios de trabajo flexibles, en especial, cuando existen compromisos familiares o asuntos personales, en lugar de promover que sus colaboradores sacrifiquen los asuntos familiares importantes por el trabajo. En la faceta informacional, la relación parece explicarse por la vía de valorar una comunicación abierta, confiable y honesta, mientras que podría verse afectada de forma negativa cuando el colaborador percibe - como injusto- que solo un grupo de personas en la organización - p. ej., los favoritos del jefe- manejen información relevante de interés general - p. ej., posibles periodos de vacaciones o futuros cambios en los turnos de trabajo- 
Finalmente, resulta importante destacar que la investigación ha demostrado que los estilos de liderazgo de los supervisores, percibidos como injustos por sus colaboradores, con procedimientos $\mathrm{y}$ tratos inadecuados o desiguales, generan estados emocionales negativos tales como estrés, frustración y ansiedad, estados que suelen vincularse a la existencia de conflicto trabajo-familia (Malisetty y Kumari, 2016; Kyei-Poku, 2014). En ese orden de ideas, la relación entre el estilo de liderazgo del jefe y el nivel de conflicto trabajo-familia del colaborador no se presenta de manera directa, debido a que la tensión en el colaborador la genera, precisamente, la percepción de injusticia. Caso contrario sucede cuando el líder comprende y trata de manera individual a cada uno de sus seguidores y los estimula a buscar alternativas de solución a sus problemas, como, por ejemplo, llamadas a la casa o jornadas flexibles que les permitan atender compromisos familiares. Estas acciones contribuyen a disminuir los niveles de conflicto trabajo-familia.
En síntesis, la revisión de la literatura sugiere que los diferentes atributos del liderazgo generan situaciones en la organización que los colaboradores perciben como justas/injustas y, como se mencionó, de tales percepciones parecen desprenderse afectaciones sobre los niveles de conflicto trabajo-familia que llegan a exhibir sus colaboradores. De acuerdo con lo anterior, se plantean las siguientes hipótesis de trabajo:

- Hipótesis 1. La percepción de justicia interaccional que los colaboradores tienen de su jefe se encuentra relacionada de forma negativa con el nivel de conflicto trabajo-familia de los colaboradores.

- Hipótesis 2. La percepción de justicia procedimental que los colaboradores tienen de su jefe se encuentra relacionada de forma negativa con el nivel de conflicto trabajo-familia de los colaboradores.

\section{Metodología}

$\mathrm{L}$ a metodología se presenta en cuatro subsecciones: en la primera se describe el tipo y corte de la investigación; en la segunda sección se describen los participantes en la investigación y algunas características sociodemográficas; en la tercera se detallan las variables de estudio y sus componentes, y en la cuarta el software para el análisis de la información.

\subsection{Procedimiento}

La investigación fue de carácter explicativo-causal, ya que, como lo plantean Hernández, Fernández y Baptista (2010), buscó establecer las causas de los eventos, sucesos o fenómenos que se estudian. En este caso, la investigación abordó la relación que existe entre tres variables diferentes - justicia procedimental y justicia interaccional, variables independientes; conflicto trabajo-familia, variable dependiente- $\mathrm{y}$ determinó el porqué de los hechos, con base en un análisis de las relaciones causa-efecto.

\subsection{Participantes}

Se realizó la investigación en tres organizaciones pertenecientes a diferentes sectores económicos - servicios, financiero y producción-. En total se aplicaron 450 encuestas a los empleados de las organizaciones. La muestra estuvo compuesta en un $60,5 \%$ por hombres, y en un $39,5 \%$ por mujeres. El promedio de edad fue de 35,8 años; el promedio de hijos por persona fue de 1,3 y el de adultos mayores a cargo fue de 0,5. Con respecto al nivel de educación de las personas, el 3,0 \% cuenta con estudios de primaria, el $27 \%$ de secundaria, el $28,1 \%$ en formación técnica, el 30,2 \% ha alcanzado un nivel de formación profesional, y el 11,7 \% algún tipo de formación posgradual. Finalmente, en el ámbito organizacional, los encuestados tuvieron en promedio 60,5 meses de antigüedad, el 3,0 \% pertenecía al nivel directivo, el $31,5 \%$ al administrativo y el $65,5 \%$ al nivel operativo. 


\subsection{Medidas}

En la recolección de la información se aplicó un cuestionario físico con diferentes escalas de medición como instrumento de recolección de datos. Tal como se mostrará más adelante, la confiabilidad y validez de cada una de las escalas de medición contenidas en el instrumento de recolección de información se probó a través del análisis del alfa de Cronbach y del análisis factorial confirmatorio o CFA, respectivamente. A continuación, se describen cada una de las escalas utilizadas con fin de medir las variables objeto de estudio.

\subsubsection{Variable independiente.}

La percepción de justicia organizacional por parte de los empleados encuestados se configuró como la variable independiente. Para la medición de esta variable se utilizó el instrumento Colquitt's Organizational Justice Scale o COJS, desarrollada por Colquitt (2001). Según las hipótesis planteadas, para la presente investigación se tuvieron en cuenta solo las dos subescalas correspondientes a las dimensiones procedimental (JP) e interaccional (JI) de la justicia. La escala se encuentra validada y su traducción adaptada tanto al idioma español (Díaz-Gracia, Barbaranelli y Moreno-Jiménez, 2014) como al contexto cultural y organizacional latinoamericano y colombiano (García y Forero, 2014).

La subescala justicia procedimental está compuesta por siete ítems. Algunos ejemplos son: «Usted ha podido expresar sus puntos de vista y sentimientos ante los procedimientos utilizados para dar recompensas». Mientras que la subescala justicia interaccional está compuesta por nueve ítems. Un ejemplo es: «Su jefe lo ha tratado con dignidad». Para cada uno de los ítems los encuestados indicaron su percepción en una escala tipo Likert de cinco puntos, en la que 1 significa totalmente en desacuerdo y 5 totalmente de acuerdo.

Respecto a la validez de las subescalas, estudios previos como, por ejemplo, el de Rodríguez, Martínez y Salanova (2014), presentaron índices de confiabilidad alfa adecuados. En esta investigación dichos índices fueron también buenos: para JP, $\alpha=0,90, y$ para JI, $\alpha=0,90$.

\subsubsection{Variable dependiente.}

En este caso, la variable dependiente fue el nivel de conflicto trabajo-familia que reportaron los empleados. Para la medición de la variable se utilizó el instrumento Survey Work-Home Interaction-Nijmegen o SWING de Geurts et al. (2005). Dicha escala se validó y adaptó tanto al idioma español (Moreno-Jiménez, Sanz, Rodríguez y Geurts, 2009) como al contexto cultural y organizacional latinoamericano (Romeo, Berger, Yepes-Bladó y Ramos, 2014).

Dado que el objetivo de la presente investigación fue abordar la interferencia del trabajo en la familia, se tomó solo la subescala de interacción negativa trabajo-familia, la cual contiene ocho ítems. Un ejemplo de los ítems que contiene dicha subescala es: «Tengo que cancelar planes con mi pareja/familia/ amigos debido a compromisos laborales». Para los ocho ítems los encuestados indicaron su percepción en una escala tipo Likert de cuatro puntos, en la que 1 significa nunca y 4 significa siempre. Frente a la validez de la subescala, estudios previos como el de Romeo et al. (2014) presentaron índices de confiabilidad alfa adecuados. En la presente investigación el nivel de confiabilidad para escala de conflicto trabajo-familia fue buena, $\alpha=0,89$.

\subsection{Análisis de datos}

En términos generales, a fin de detectar los efectos propuestos en las hipótesis de investigación y evaluar las relaciones y los efectos entre las variables se utilizó un modelo de ecuaciones estructurales - structural equation modeling o SEM- (Anderson y Gerbing, 1988). El SEM es una técnica estadística de análisis multivariado que permite poner a prueba relaciones causales — usando datos cuantitativos-fundamentadas en supuestos o reflexiones cualitativas sobre el sentido de la causalidad (Hair, Anderson, Tatham y Black, 1999).

El SEM permitió establecer el valor perteneciente a cada relación teórica propuesta y, más importante, 
algunos índices que expresan el grado en el que los datos se ajustan al modelo teórico propuesto, de manera que confirmaba su validez. El ajuste del modelo teórico propuesto se evaluó a través de diferentes índices de bondad de ajuste tales como el Root Mean Square Error of Aproximation o RMSEA, el cual debe estar por debajo de 0,08 para indicar un buen ajuste; el Goodness of Fit Indexo GFI, que muestra la cantidad de varianzas y covarianzas explicadas por el modelo y debe ser mayor de 0,90 para un ajuste adecuado del modelo; el Adjusted Goodness of Fit Index o AGFI, el cual debe ser superior a 0,80; y el Comparative Fit Index o CFI, el cual debe ser mayor que 0,90 para indicar bondad en el ajuste del modelo teórico propuesto.

Por último, a fin de efectuar los análisis respectivos se empleó el software Amos Graphics y SPSS, dos de los programas estadísticos más usados en el análisis de SEM en las ciencias sociales.

\section{Resultados}

$\mathrm{L}$ as estadísticas descriptivas y las correlaciones entre las variables de estudio se presentan en la tabla 1.

Tabla 1. Medias, desviaciones estándar y correlaciones de las variables del estudio

\begin{tabular}{l|c|c|c|c|c}
\hline \multicolumn{1}{c|}{ Variable } & M & DE & $\mathbf{1}$ & $\mathbf{2}$ & $\mathbf{3}$ \\
\hline 1. Justicia procedimental & 3,17 & 0,91 & $(0,90)$ & & \\
2. Justicia interaccional & 3,65 & 0,85 & $0,532^{* *}$ & $(0,90)$ & $(0,89)$ \\
3. Conflicto trabajo-familia & 1.96 & 0,66 & $0,086^{* *}$ & $-0,427^{* *}$ & \\
Índices de confiabilidad -alfa de Cronbach-son reportados en la diagonal. \\
** Correlación significativa al 0,01 (bilateral)
\end{tabular}

Fuente. Elaboración propia.

\subsection{Análisis factorial confirmatorio}

Se realizó el análisis factorial confirmatorio o AFC del modelo de la variable latente justicia organizacional, ya que se trata de una variable multidimensional; es decir, está compuesta de diferentes dimensiones. A la variable de conflicto trabajo-familia no es necesario realizarle dicho análisis, en la medida en que, desde el punto de vista teórico, es una variable unidimensional.
Los índices de bondad de ajuste de la justicia organizacional con tres dimensiones fueron aceptables (véase la tabla 2), dado que algunos autores (p. ej., Bies, 2001) han propuesto que las dimensiones de justicia interpersonal e informacional realmente representan una sola dimensión de la justicia organizacional, denominada «justicia interaccional» $\mathrm{o}$ JI. 
Tabla 2. Índices de ajuste de los modelos de AFC de la justicia organizacional

\begin{tabular}{lccc}
\hline \multicolumn{1}{c|}{ Índice } & Estimación del modelo & Nivel de aceptación \\
\hline Chi-cuadrado & 551,368 & $\mathrm{P}<0,00$ \\
Gl & 161 & \\
CMIN/DF & 3,426 & $<3,00$ \\
CFI & 0,940 & $>0,90$ \\
TLI & 0,930 & $>0,90$ \\
IFI & 0,940 & $>0,90$ \\
RFI & 0,904 & $>0,90$ \\
NFI & 0,917 & $>0,90$ \\
GFI & 0,892 & $>0,90$ \\
AGFI & 0,860 & $>0,80$ \\
RMSEA & 0,072 & $\leq 0,08$ \\
\hline
\end{tabular}

Fuente: Elaboración propia.

\subsection{Análisis del modelo estructural y prueba de las hipótesis}

En la tabla 3 se muestran los valores de los diferentes índices de bondad de ajuste para el modelo estructural a través de los cuales se analizaron las relaciones propuestas y, por tanto, se pusieron a prueba las hipótesis de investigación. Al respecto, el modelo obtuvo un buen ajuste a los datos.

Tabla 3. Índices de ajuste del modelo estructural teóricamente propuesto

\begin{tabular}{ccc}
\hline Índices & Estimación del modelo & Nivel de aceptación \\
\hline Chi-cuadrado & 952,557 & $\mathrm{P}<0,00$ \\
Gl & 341 & \\
CMIN/DF & 2,793 & $<3,00$ \\
CFI & 0,928 & $>0,90$ \\
TLI & 0,921 & $>0,90$ \\
IFI & 0,929 & $>0,90$ \\
RFI & 0,882 & $>0,90$ \\
NFI & 0,893 & $>0,90$ \\
GFI & 0,875 & $>0,90$ \\
AGFI & 0,851 & $>0,80$ \\
RMSEA & 0,062 & $\leq 0,08$ \\
\hline
\end{tabular}

Fuente: Elaboración propia. 
El modelo propuesto contiene la relación entre la justicia procedimental, la justicia interaccional y el conflicto trabajo-familia. Como se puede observar en la figura 1, solo la justicia interaccional tuvo una relación negativa y significativa con el conflicto trabajo-familia —0,427; p < 0,00—; por su parte, la relación justicia procedimental y conflicto trabajo-familia no fue significativa $-0,086 ; \mathrm{p}<0,00-$. Así, entonces, los datos correspondientes al modelo ofrecen soporte para la hipótesis 2, pero no ofrecen soporte para la hipótesis 1. Estos resultados se discuten a continuación.

Figura 1. Resultados del modelo de ecuaciones estructurales

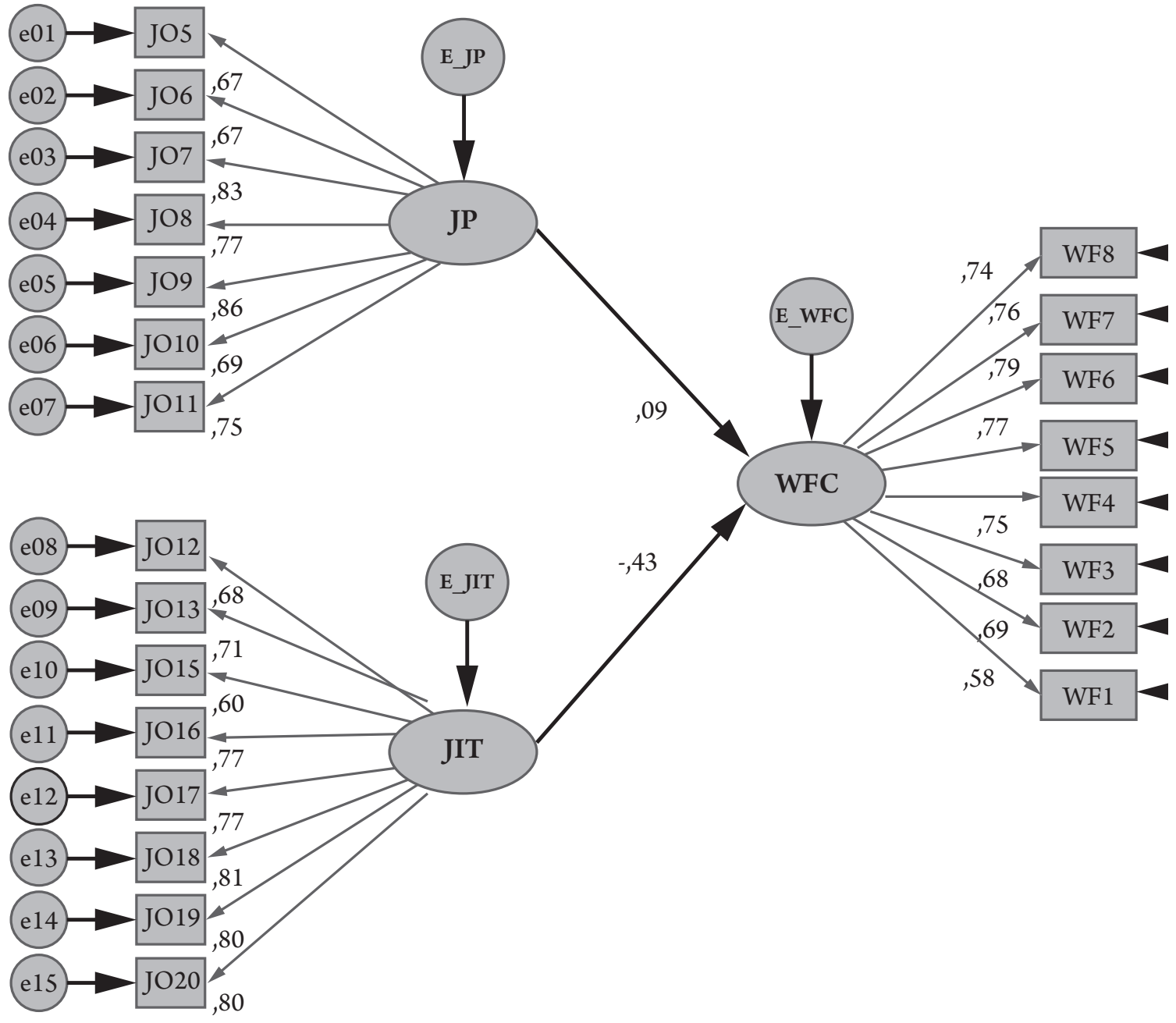

Fuente. Elaboración propia. 


\section{Discusión}

as evidencias encontradas contribuyen a las
Jinvestigaciones y plantean nuevas perspectivas que permiten comprender la relación entre el liderazgo transformacional y el conflicto trabajo-familia en las organizaciones, con una serie de implicaciones prácticas y teóricas. En primer lugar, la investigación reveló que la justicia organizacional se encuentra relacionada de forma negativa con el conflicto trabajo-familia. En el caso de la presente investigación, se analizó el rol mediador de las dimensiones procedimental (JP) e interaccional (JI) de la justicia.

La justicia interaccional se relacionó de forma significativa y negativa con el conflicto trabajo-familia. Estos resultados están en consonancia con los hallazgos de Judge y Colquitt (2004), y Kyei-Poku (2014). Por su parte, a diferencia de lo esperado, la relación entre la justicia procedimental y el conflicto trabajo-familia no fue significativa, pero fue positiva. La aparentemente contradictoria relación positiva entre justicia procedimental y conflicto trabajo-familia podría entenderse desde la perspectiva de la teoría del intercambio social (Blau, 1964). Esto, en la medida en que los empleados que perciben los procedimientos implementados por la organización como justos, como, por ejemplo, la política de toma de decisiones participativa, podrían estar inclinados a «devolver el favor» a la organización por medio de actitudes positivas hacia esta, tales como el compromiso organizacional (Grant, Dutton y Rosso, 2008). Así, basado en la norma de reciprocidad (Gouldner, 1960), el tratamiento positivo por parte de la organización genera un sentimiento de endeudamiento, por lo cual el empleado puede llegar a dedicar tiempo destinado a la familia a las labores de la organización. Esto sucede especialmente con la justicia procedimental, debido a que, como se anotó, los colaboradores valoran esta dimensión como un aspecto estructural en la organización (Siegel, Post, Brockner, Fishman y Garden, 2005) y la posibilidad de una relación en el largo plazo (Judge y Colquitt, 2004), por lo cual la percepción de compromiso es mayor.

Así, la justicia interaccional representa el mecanismo psicológico a través del cual el estilo de liderazgo de los supervisores termina por afectar —al disminuirlos niveles de conflicto que los colaboradores exhiben entre el trabajo y su familia. Es decir, el hecho de que los líderes brinden apoyo a sus empleados, respondan a sus necesidades personales y los ayuden a pensar de forma creativa cuando tienen que enfrentar situaciones contradictorias o problemáticas (Grzywacz y Marks, 2000) genera en el colaborador percepciones de justicia en el trato que parecen ayudarle a manejar mejor la relación entre el trabajo y la familia, o, por lo menos, no parecen percibir que su trabajo interfiera con su vida familiar, pues conciben dicho trabajo como justo. El buen trato en el trabajo se traslada a la familia por la vía del efecto psicológico que generan las percepciones de justicia asociada a dicho trato.

Como lo han destacado investigaciones previas, se torna vital para las organizaciones tratar de minimizar los niveles de conflicto entre el trabajo y la familia, pues dichos niveles de conflicto suelen estar relacionado de forma positiva con fenómenos contraproducentes como lo son el estrés y el agotamiento de los empleados (Amstad, Meier, Fasel, Elfering y Semmer 2011), su pérdida de salud física, así como promover en estos menores niveles de satisfacción laboral y mayores niveles de rotación (O’Neill y Davis, 2011). 


\section{Conclusiones}

$\mathrm{E}$ sta investigación destaca el valor de la percepción de la justicia organizacional, en su dimensión interaccional, en la disminución de los niveles de conflicto entre el trabajo y la familia. Esto, en la medida en que la correlación entre justicia interaccional y conflicto trabajo-familia fue significativa y negativa, mientras que la justicia procedimental no fue significativa.

Desde los hallazgos de esta investigación se apunta a especialistas en recursos humanos para que trabajen en programas de desarrollo de competencias de jefes o supervisores en una organización, a fin de que tengan un mejor trato con sus colaboradores o subordinados y les brinden respeto, honestidad, dignidad y cortesía. Lo anterior en cuanto es este tipo de trato o interacción el que ayuda a generar en los empleados percepciones de justicia y, por esta vía, contribuye a disminuir los niveles de conflicto trabajo-familia que estos últimos presentan.

La dimensión interaccional de la justicia organizacional representa el mecanismo psicológico por medio del cual el comportamiento del líder contribuye a disminuir los niveles de conflicto de los empleados. En la medida en que los colaboradores perciban tratos dignos, suministro de información confiable y solidaridad en la búsqueda de soluciones a sus inconvenientes, disminuirá la probabilidad de que experimenten fenómenos asociados al estrés y el agotamiento emocional.

\section{Referencias}

Amstad, F. T.; Meier, L. L.; Fasel, U.; Elfering, A.; Semmer, N. K. (2011). A meta-analysis of work-family conflict and various outcomes with a special emphasis on cross-domain versus matching-domain relations. Journal of Occupational Health Psychology, 16, 151-169. DOI: https://doi.org/10.1037/ $\underline{\mathrm{a} 0022170}$

Anderson, J. C.; Gerbing, D. W. (1988). Structural equation modeling in practice: a review and recommended two-step approach. Psychological Bulletin, 103, 411-423. DOI: https:// doi.org/10.1037/0033-2909.103.3.411

Bies, R. J. (2001). International (in)justice: the sacred and the profane. En J. Greenberg y R. Cropanzano (Eds.), Advances in organization justice (89-118). Palo Alto, CA: Stanford University Press.

Blau, P. M. (1964). Exchange and power in social life. Nueva York: Wiley.

Colquitt, J. (2001). On the dimensionality of organizational justice: a construct validation of a measure. Journal of Applied Psychology, 86, 386-400. DOI: https://doi. org/10.1037/0021-9010.86.3.386

Colquitt, J.; Zipay, K. (2015). Justice, fairness, and employee reactions. Annual Review of Organizational Psychology and Organizational Behavior, 2, 1-25. DOI: https://doi. org/10.1146/annurev-orgpsych-032414-111457
Cropanzano, R.; Bowen, D. E.; Gilliland, S. W. (2007). The management of organizational justice. Academy of Management Perspectives 21, 34-48. DOI: https://doi.org/10.5465/ amp.2007.27895338

Cropanzano, R.; Byrne, Z. S.; Bobocel, D. R.; Rupp, D. E. (2001). Moral virtues, fairness heuristics, social entities, and other denizens of organizational justice. Journal of Vocational Behavior, 58, 164-209. DOI: https://doi.org/10.1006/ jvbe.2001.1791

Cropanzano, R.; Prehar, C. A.; Chen, P. Y. (2002). Using social exchange theory to distinguish procedural from interactional justice. Group and Organizational Management, 27, 324-351. https://doi.org/10.1177/1059601102027003002

De Cremer, D.; Van Dijke, M. H.; Bos, A. E. R. (2007). When leaders are seen as transformational: the effects of organizational justice. Journal of Applied Social Psychology, 37(8), 1797-1816. DOI: https://doi.org/10.1111/j.15591816.2007.00238.x

Díaz-Gracia, L.; Barbaranelli, C.; Moreno-Jiménez, B. (2014). Spanish version of Colquitt's Organizational Justice Scale. Psicothema, 26(4), 538-544.

Ferris, D. L.; Spence, J. R.; Brown, D. J.; Heller, D. (2012). Interpersonal deviance and workplace deviance: the role of esteem threat. Journal of Management, 38, 1788-1811. DOI: https://doi.org/10.1177/0149206310372259 
Folger, R. (2001). Fairness as Deonance. En Gilliland, S.; Steiner, D.; Skarlicki, D (Eds.), Theoretical and Cultural Perspectives on Organizational (3-33). Greenwich: Information Age Publishing.

García, M.; Forero, C. (2014). Cambio organizacional y percepción de la justicia organizacional en una entidad de la ciudad de Bogotá-Colombia. Diversitas: Perspectivas en Psicología, 10(2), 93-305. DOI: https://doi.org/10.15332/ $\underline{\mathrm{s} 1794-9998.2014 .0002 .07}$

Geurts,S.; Taris, T.W.; Kompier,M.; Dikkers,J.S.; van Hooff,M.L.; Kinnunen, U. M. (2005). Work-home interaction from a work psychological perspective: development and validation of a new questionnaire, the SWING. Worky Stress, 19(4), 319-339. DOI: $\underline{\text { https://doi.org/10.1080/02678370500410208 }}$

Gouldner, A. W. (1960). The norm of reciprocity: a preliminary statement. American Sociological Review, 25, 161-78. DOI: https://doi.org/10.2307/2092623

Gillet, N.; Fouquereau, E.; Bonnaud-Antignac, A.; Mokounkolo, R.; Colombat, P. (2013). The mediating role of organizational justice in the relationship between transformational leadership and nurses' quality of work life: a cross-sectional questionnaire survey. International Journal of Nursing Studies, 50(10), 1359-1367. DOI: https://doi.org/10.1016/j. inurstu.2012.12.012

Grandey, A.; Cordeiro, B.; Judd, M. (2007). Work-family supportiveness perceptions: important for the well-being of male blue-collar hourly workers? Journal of Vocational Behavior, 71(3), 460-478. DOI: https://doi.org/10.1016/j. jub.2007.08.001

Grant, A.; Dutton, J.; Rosso, B. (2008). Giving commitment: employee support programs and the prosocial sensemaking process. Academy of Management Journal, 51, 898-918. DOI: https://doi.org/10.5465/amj.2008.34789652

Greenberg, J. (1990). Organizational justice: yesterday, today, and tomorrow. Journal of Management, 16(2), 399-432. DOI: https://doi.org/10.1177/014920639001600208

Greenberg, J. (1993). Stealing in the name of justice: informational and interpersonal moderators of theft reactions to underpayment inequity. Organizational Behavior and Human Decision Processes, 54, 81-103. DOI: https://doi. org/10.1006/obhd.1993.1004

Greenberg, J.; Colquitt, J. A. (2005). Handbook of organizational justice. Mahwah, Nueva Jersey: Lawrence Erlbaum Associates.

Greenhaus, J. H.; Beutell, N.J. (1985). Sources of conflict between work and family roles. Academy of Management Review, 10, 76-88. DOI: https://doi.org/10.5465/amr.1985.4277352

Grzywacz, J. G.; Marks, N. F. (2000). Reconceptualizing the work-family interface: an ecological perspective on the correlates of positive and negative spillover between work and family. Journal of Occupational Health Psychology, 5(1), 111-126. DOI: https://doi.org/10.1037/1076-8998.5.1.111

Hair, J.; Anderson, R.; Tatham, R.; Black, W. (1999). Análisis multivariante ( $\left.5^{\mathrm{a}} \mathrm{ed}.\right)$. Madrid: Prentice Hall. DOI: https:// doi.org/10.1080/1478601X.2016.1167054
Hanif, E. G.; Lambert, L. D. K.; James, F. (2016). Exploring the association between organizational structure variables and work on family strain among Indian police officers, Criminal Justice Studies, 29(3), 253-271.

Hernández, R.; Fernández, C.; Baptista, M. P. (2010). Metodología de la investigación ( $5^{\mathrm{a}}$ ed.). México: McGraw-Hill Educación.

Jiang, H. (2012). A model of work-life conflict and quality of employee-organization relationships (EORs): transformational leadership, procedural justice, and family-supportive workplace initiatives. Public Relations Review, 38, 231-245. DOI: https://doi.org/10.1016/j. pubrev.2011.11.007

Judge, T. A.; Colquitt, J. A. (2004). Organizational justice and stress: the mediating role of work-family conflict. Journal of Applied Psychology, 89(3), 395-404. DOI: https://doi. org/10.1037/0021-9010.89.3.395

Kim, H.; Kim, J. A. (2015). Cross-level study of transformational leadership and organizational affective commitment in the Korean local governments: mediating role of procedural justice and moderating role of culture types based on competing values framework. Leadership, 11(2) 158-185. DOI: $\underline{\text { https://doi.org/10.1177/1742715013514880 }}$

Kirkman, B. L.; Chen, G.; Farh, G. L.; Chen, Z. J.; Lowe, K. B. (2009). Individual power distance orientation and follower reactions to transformational leaders: a cross-level, cross-cultural examination. Academy of Management Journal, 52, 744-764. DOI: https://doi.org/10.5465/ amj.2009.43669971

Kossek, E.; Pichler, S.; Bodner, T.; Hammer, L. (2011). Workplace social support and work-family conflict: a meta-analysis clarifying the influence of general and work-family-specific supervisor and organizational support. Personnel Psychology, 64, 289-313. DOI: https://doi.org/10.1111/j.17446570.2011.01211.x

Kossek, E.; Ozeki, C. (1998). Work-family conflict, policies, and the job-life satisfaction relationship: a review and directions for organizational behavior-human resources research. Journal of Applied Psychology, 83, 139-149. DOI: https://doi. org/10.1037/0021-9010.83.2.139

Kyei-Poku, I. A. (2014). Linking interactional justice to work-tofamily conflict: the mediating role of emotional exhaustion. Organization Management Journal, 11(2), 74-83. DOI: https://doi.org/10.1080/15416518.2014.929932

Leventhal, G. S. (1976). Justice in social relationships. En J. W. Thibaut; J. T. Spence; R. C. Carson (Eds.), Contemporary topics in social psychology (211-240). Morristown, NJ: General Learning Press.

Leventhal, G. S. (1980). What should be done with equity theory? New approaches to the study of justice in social relationships. En K. Gergen; M. Greenberg; R. Willis (Eds.), Social exchange: advances in experimental and social psychology (Vol. 9, 91-131). Nueva York: Plenum. DOI: https://doi.org/10.1007/978-1$\underline{4613-3087-5 \quad 2}$

Lind, E. A.; Tyler, T. R. (1988). The social psychology of procedural justice. Nueva York: Plenum. DOI: https://doi. org/10.1007/978-1-4899-2115-4 
Luo, Y. (2007). The independent and interactive roles of procedural, distributive, and interactional justice in strategic alliances. Academy of Management Journal, 50, 644-664. DOI: https://doi.org/10.5465/amj.2007.25526452

Malisetty, S.; Kumari, K. V. (2016). An investigation on relationship of deviance workplace behavior with organisational justice, abusive supervision and work-family conflict. Indian Journal of Science and Technology, 9(39), 1-13. DOI: https://doi.org/10.17485/ijst/2016/v9i39/100449

Moreno-Jiménez, B.; Sanz, A. I.; Rodríguez, A.; Geurts, S. (2009). Propiedades psicométricas de la versión española del Cuestionario de Interacción Trabajo-Familia (SWING). Psicothema, 21(2), 331-337.

Munir, F.; Nielsen, K.; Garde, A. H.; Albertsen, K.; Carneiro, I. G. (2012). Mediating the effects of work-life conflict between transformational leadership and health-care workers job satisfaction and psychological wellbeing. Journal of Nursing Management, (20), 512-521. DOI: https://doi.org/10.1111/ j.1365-2834.2011.01308.x

O’Neill, J. W.; Davis, K. (2011). Work stress and well-being in the hotel industry. International Journal of Hospitality Management, 30, 385-390. DOI: https://doi.org/10.1016/j. ijhm.2010.07.007

Romeo, M.; Berger, R.; Yepes-Baldó, M.; Ramos, B. (2014). Adaptation and validation of the Spanishversion of the «Survey Work-Home Interaction-NijmeGen» (SWING) to Spanish speaking countries. Anales de Psicología, 30(1), 287-293. DOI: https://doi.org/10.6018/analesps.30.1.148291

Rodríguez, R. L.; Martínez, M.; Salanova, M. (2014). Justicia organizacional, engagement en el trabajo y comportamientos de ciudadanía organizacional: una combinación ganadora. Universitas Psychologica, 13(3), 961-974. DOI: https://doi. org/10.11144/Javeriana.UPSY13-3.joet

Siegel, P. A.; Post, C.; Brockner, J.; Fishman, A.; Garden, C. (2005). The moderating influence of procedural fairness on the relationship between work-life conflict and organizational commitment. Journal of Applied Psychology, 90(1), 13-24. DOI: https://doi.org/10.1037/0021-9010.90.1.13
Taner, B.; Turhan, M.; Helvaci, I.; Köprülü, O. (2015). The effect of the leadership perception and organizational justice on organizational commitment: a research in a State university. International Review of Management and Marketing, 5(3), 180-194.

Thibaut, J.; Walker, L. (1975). Procedural justice: a psychological analysis. Hillsdale, NJ: Erlbaum.

Tyler, T. R.; Blader, S. L. (2000). Cooperation in groups: procedural justice, social identity, and behavioral engagement. Philadelphia: Psychology Press.

Tyler, T. R.; Smith, H. (1998). Social justice and social movements. En D. Gilbert; S. Fiske; G. Lindzey (Eds.), Handbook of Social Psychology (4a ed., vol. 2, 595-629). Nueva York: McGraw-Hill.

Tziner, A.; Sharoni, G. (2014). Organizational citizenship behavior, organizational justice, job stress, and work-family conflict: examination of their interrelationships with respondents from a non-Western culture. Journal of Work and Organizational Psychology, 30, 35-42. DOI: https://doi. org $/ 10.5093 / \operatorname{tr} 2014 \mathrm{a} 5$

Weiss, H. M.; Suckow, K.; Cropanzano, R. (1999). Effects of justice conditions on discrete emotions. Journal of Applied Psychology, 84,786-794.DOI: https://doi.org/10.1037/00219010.84.5.786

Williams, K. J.; Alliger, G. M. (1994). Role stressors, mood spillover, and perceptions of workfamily conflict in employed parents. Academy of Management Journal, 37(4), 837-868. DOI: $\underline{\text { https: //doi.org/10.2307/256602 }}$

Zeinabadi, H.; Rastegarpour, H. (2010). Factors affecting teacher trust in principal: testing the effect of transformational leadership and procedural justice. Procedia Social and Behavioral Sciences, 5, 1004-1008. DOI: https://doi. org/10.1016/j.sbspro.2010.07.226 
